\title{
Mixed Consistency Model: Meeting Data Sharing Needs of Heterogeneous Users*
}

\author{
Zhiyuan Zhan \\ College of Computing \\ Georgia Institute of Technology \\ USA \\ zzhan@cc.gatech.edu
}

\author{
Mustaque Ahamad \\ College of Computing \\ Georgia Institute of Technology \\ USA \\ mustaq@cc.gatech.edu
}

\author{
Michel Raynal \\ IRISA \\ University of Rennes \\ France \\ raynal@irisa.fr
}

\begin{abstract}
Heterogeneous users usually have different requirements as far as consistency of shared data is concerned. This paper proposes and investigates a mixed consistency model to meet this heterogeneity challenge in large scale distributed systems that support shared objects. This model allows combining strong (Sequential) consistency and weak (Causal) consistency. The paper defines the model, motivates it and proposes a protocol implementing it.
\end{abstract}

\section{Introduction}

Context: heterogeneous users The rapid proliferation of mobile devices and wireless networks has made it possible to access and share information across different platforms with varying degree of computing power and network resources. For example, various cars can share traffic information through on-board communication devices. This scenario requires that the information be shared, disseminated and updated at a potentially large number of heterogeneous users. There are two sources of heterogeneity in such an environment: user needs and system resources. For instance, some users may not care about each new update, while others do. Also, some users can use relatively powerful systems to access the information, while others may only be able to use wireless handheld device to do so. The lack of computing power and/or network resources will prevent them from employing expensive protocols to ensure high quality of shared information at all time. Both sources suggest that the information sharing system should take heterogeneity as a primary concern if it is to meet the needs of varied users in a wide area network.

Introducing a mixed consistency model We assume that shared information is encapsulated in distributed objects.

\footnotetext{
*This work was supported in part by NSF grant ITR-0121643.
}

Object replication is a common technique to increase the scalability and robustness of such systems, which also introduces the consistency problem. Many consistency models have been proposed in recent years. The tradeoff between performance and the level of consistency presents a dilemma. For example, strong consistency such as sequential consistency ensures an unique order of operations across different replicas, but its implementation is costly and does not scale very well; the ordering guarantees provided by different weak consistency models may result in conflicting views of shared critical information but they are relatively easy to implement with good performance. Therefore, only supporting a single level of consistency, which is commonly done in existing systems, either does not scale well or is insufficient for meeting user requirements. In order to maintain consistency in a heterogeneous environment, we are exploring an approach of employing mixed consistency for objects which addresses both user needs and system resource constraints.

- For a particular piece of information, users can choose the consistency level they desire based on the current resource level they have. Resourceful users can access information with stronger consistency, while others may be forced to access information with weaker consistency guarantees due to lack of resources.

- Strong consistency for critical information is necessary but has a relatively high maintenance cost. In many applications, weaker consistency provides a relatively cheap yet scalable way for a large number of users to share information that has less stringent correctness requirements.

With a mixed consistency model, applications can make use of both high (strong) and low (weak) levels of consistency to meet their needs based on the availability of resources at various nodes. However, mixing leads to a new problem: how to meaningfully define consistency when resource poor nodes want to access an object replica in a 
weak mode whereas others want to maintain strong consistency for their replicas of this same object. Operations observed by strong replicas should appear to be executed on a single copy serially, but nodes with weak replicas do not have the resources necessary to ensure such strong ordering. Since update operations produce new values for replicas and strong replicas must observe new values in a consistent order, we identify constraints that prohibit values of updates done at weak replicas to be disseminated to strong replicas in our model. At the same time, a weak replica can observe values of updates done at strong replicas as well as at other weak replicas. Such a model can meet the needs of many applications. In order to bound the difference between weak and strong replicas, weak replicas can periodically update themselves with values written by nodes that maintain strong replicas. The dissemination flow constraints allow us to precisely characterize the mixed consistency model, and they can easily be incorporated into a protocol that implements this model in a heterogeneous environment.

Although the mixed consistency model we propose is not limited to any particular consistency models, sequential consistency (SC) and causal consistency (CC) are the strong and weak consistency models we consider in this paper. They both have been extensively studied over the past years and many protocols have been proposed to implement them. We use consistency tags associated with replicas and processes to implement the constraints needed in a mixed consistency model.

- Each object replica has a consistency tag of either SC or CC. SC replicas encapsulate critical information updates that should be maintained under sequential consistency. CC replicas encapsulate other information which is only maintained under causal consistency.

- Each process has a consistency tag of either SC or CC. $\mathrm{SC}$ processes are resourceful processes that can afford to use more expensive protocols to access different types of information, while $\mathrm{CC}$ processes are resource limited processes that can only afford cheap protocols for the same information.

Paper contributions A careful study of mixed consistency leads to a rich set of possibilities. In this paper, we formally define a mixed consistency model to generalize many application scenarios, where each object can have both SC and $\mathrm{CC}$ replicas at the same time. We also propose a protocol to ensure both SC and CC consistency requirements. The main contributions of this paper are:

- Based on different application requirements, we propose a unified mixed consistency model allowing both $\mathrm{SC}$ and $\mathrm{CC}$ replicas to coexist at the same time.
- We design a mixed consistency protocol to ensure both SC and CC at the same time and show that it is correct and can offer performance versus consistency tradeoff in a heterogeneous environment.

The rest of the paper is organized as follows: Section 2 introduces the system model. Section 3 formally defines the mixed consistency model. Section 4 provides a mixed consistency protocol implementing the model and outlines the correctness proof. Section 5 presents performance related analysis of our protocol. Section 6 discusses the related work. Section 7 finally summarizes the paper and lists future work.

\section{System Model}

We consider a replicated object system composed of distributed processes, each of which having a local memory where copies of objects are stored. We assume that one process can only have at most one replica of a particular object in its local memory. Replicas can be removed from the local memory when they are no longer needed or there is a memory shortage. New object replicas can be created as needed. However, we will not discuss the creation and deletion of replicas in this paper. We will focus our discussion on a system where the locations of object replicas are already determined. In such a system, a process has access to its local copies of objects. Processes can also communicate with each other through reliable pair wise inter-process communication channels.

Let $\mathrm{P}$ denote the process set, where each $p_{i} \in P$ represents an individual process (assuming $1 \leq i \leq N$ ). We define that $\mathrm{P}$ consists of two disjoint subsets: $P_{S C}$ and $P_{C C}$. Processes in $P_{S C}\left(P_{C C}\right)$ are called SC (CC) processes.

Let $\mathrm{O}$ denote the object replica set, where each $o_{i j} \in O$ stands for a replica of object $o_{j}$ stored in process $p_{i}$ 's local memory (assuming $1 \leq j \leq M$ ). Clearly for a particular $j$ $(1 \leq j \leq M)$, all the $o_{x j} \in O(1 \leq x \leq N)$ are initialized from the same object $o_{j}$. Similarly, we define that $\mathrm{O}$ also consists of two disjoint subsets: $O_{S C}$ and $O_{C C}$. Replicas in $O_{S C}\left(O_{C C}\right)$ are called $\mathrm{SC}(\mathrm{CC})$ replicas.

\section{The Mixed Consistency Model}

A process $p_{i}$ accesses an object $o_{j}$ by invoking the methods on an object replica $o_{i j}$ in its local memory. We categorize the methods into "read" (r) and "write" (w) operations. The "read" method does not change the state of the object copy, while the "write" method does. We adopt the notation used in paper [2] to define the consistency model of our system, i.e., operation $r_{i}\left(o_{j}\right) v$ stands for $p_{i}$ reading object $o_{j}$ and returning its current value (or state) $v$, and operation $w_{i}\left(o_{j}\right) v$ stands for $p_{i}$ writing to object $o_{j}$ a new value $v$. We 
also use $r\left(o_{j}\right) v$ and $w\left(o_{j}\right) v$ to denote the operations when who issues the operation is clear or unimportant.

\subsection{Access Constraints}

Mixed consistency aims to meet both application needs and resource limitations that are common in heterogeneous environments. Thus, when a resource poor node chooses to make a weak replica of an object and updates it, it cannot be expected to have enough resources to update the strong replicas of the object at other nodes in a manner that is required by the $\mathrm{SC}$ model. On the other hand, SC requires that updates observed by strong replicas must be ordered in a uniform manner. These conflicting goals must be reconciled in the mixed consistency model such that strong replicas attain the desired type of ordering for operations observed by them, while allowing weak replicas to achieve the sharing and efficiency required by them. We achieve these goals by developing a set of access constraints that allow weak replicas to access the values of updates done at strong as well as weak replicas. Strong replicas can only observe values of those updates that are done at the nodes that maintain strong replicas. This is reasonable because (1) nodes with strong replicas have the necessary resources to enforce the strong ordering, and (2) updates done to weak replicas at resource poor nodes, which do not want to incur the cost of strongly ordering the updates, are not observed by the strong replicas to prevent violations of ordering requirements. This is also consistent with the requirements of applications where nodes with weak replicas either do not update objects that have other strong replicas or values of their updates are only shared with other nodes that have weak replicas. The constraints that define which updates can be observed by which type of replicas can be captured by the following two rules:

- Rule 1: A SC process can read and write a SC replica and can only write a $\mathrm{CC}$ replica;

- Rule 2: A CC process can read and write a CC replica and can only read a SC replica.

Table 1 defines both rules in the mixed consistency model. Each row in Table 1 gives the legal access rights of the particular process group to different object groups.

\begin{tabular}{||c|c|c||}
\hline & $O_{S C}$ & $O_{C C}$ \\
\hline$P_{S C}$ & $\mathrm{RW}$ & $\mathrm{W}$ \\
$P_{C C}$ & $\mathrm{R}$ & $\mathrm{RW}$ \\
\hline
\end{tabular}

\section{Table 1. Access Constraints Table}

\subsection{Well-formed Serialization and History}

Well-formed Serialization: Let T be an arbitrary set of operations. We say $\mathrm{T}$ is well-formed if and only if no operation in $\mathrm{T}$ violates Table 1 . We call $\mathrm{S}$ a serialization of
$\mathrm{T}$ if $\mathrm{S}$ is a linear sequence containing exactly the operations of $\mathrm{T}$ such that each read operation from an object returns the value written by the most recent write to that object. From now on, when we say a serialization, it means a serialization of a well-formed operation set by default. In other words, a serialization always respects Rule 1 and Rule 2.

For example, let's consider a system setting of two processes $\left(p_{1}, p_{2}\right)$ and three objects $\left(o_{1}, o_{2}\right.$ and $\left.o_{3}\right)$. Both $p_{1}$ and $p_{2}$ have a local copy of all three objects. $p_{1}, o_{11}, o_{22}$ are marked as SC, while $p_{2}, o_{21}, o_{12}, o_{13}, o_{23}$ are marked as $\mathrm{CC}$. We have three operation sets defined as:

$T_{1}=\left\{w_{1}\left(o_{3}\right) 1, r_{1}\left(o_{1}\right) 1, r_{1}\left(o_{2}\right) 1, r_{1}\left(o_{3}\right) 1, w_{2}\left(o_{1}\right) 2, w_{2}\left(o_{2}\right) 2\right.$, $\left.w_{2}\left(o_{3}\right) 2, r_{2}\left(o_{2}\right) 2\right\}$

$T_{2}=\left\{w_{1}\left(o_{1}\right) 1, w_{1}\left(o_{2}\right) 1, w_{2}\left(o_{3}\right) 2, r_{2}\left(o_{1}\right) 1, r_{2}\left(o_{1}\right) 2, r_{2}\left(o_{3}\right) 2\right\}$

$T_{3}=\left\{w_{1}\left(o_{1}\right) 1, r_{2}\left(o_{1}\right) 1, w_{1}\left(o_{2}\right) 2, r_{2}\left(o_{3}\right) 2, w_{2}\left(o_{3}\right) 2, r_{1}\left(o_{1}\right) 1\right\}$

We can see that $T_{1}$ is not well-formed because $r_{1}\left(o_{2}\right) 1, r_{1}\left(o_{3}\right) 1, w_{2}\left(o_{2}\right) 2$ violates Table $1 . \quad T_{2}$ is well-formed but it does not have a serialization because $r_{2}\left(o_{1}\right) 2$ returns a value 2 that has never been written to $o_{1}$ (assuming each object has an initial value 0 ). $T_{3}$ is well-formed and

$S=\left\{w_{2}\left(o_{3}\right) 2, w_{1}\left(o_{1}\right) 1, w_{1}\left(o_{2}\right) 2, r_{2}\left(o_{1}\right) 1, r_{2}\left(o_{3}\right) 2, r_{1}\left(o_{1}\right) 1\right\}$

can be one serialization of $T_{3}$.

Projection: Let $T$ be an arbitrary set of operations. We define a projection of $T$ on an arbitrary process group $S$, denoted as $T_{S}$, to be a subset of $T$, which only contains the operations performed by processes in $S$. Similarly, we also define a projection of $T$ on an arbitrary object replica group $J$, denoted as $T^{J}$, to be a subset of $T$, which only contains the operations performed to replicas in $J$. It is easy to see that $\left(T_{S}\right)^{J}=\left(T^{J}\right)_{S}=T_{S}^{J}$.

Causal Order: Let $A$ be a complete set of all operations. We define the program order $\rightarrow$ on $A$ to be a complete set of $<o p_{1}, o p_{2}>$ such that both $o p_{1}$ and $o p_{2}$ are performed by the same process $p$ and $o p_{1}$ precedes $o p_{2}$ according to $p$ 's local clock. In this case, we write $o p_{1} \rightarrow o p_{2}$.

Without loss of generality, we assume that all writes to the same object are uniquely valued. The writes-into order $\mapsto$ on $A$ is defined as such that $o p_{1} \mapsto o p_{2}$ holds if and only if there are $o_{j}$ and $v$ such that $o p_{1}=w\left(o_{j}\right) v$ and $o p_{2}=$ $r\left(o_{j}\right) v$.

A causal order $\Rightarrow$ induced by both $\rightarrow$ and $\mapsto$ in our model is a partial order that is the transitive closure of the program order and the writes-into order defined on $A$. To be specific: $o p_{1} \Rightarrow o p_{2}$ holds if and only if one of the following cases holds:

- $o p_{1} \rightarrow o p_{2}$ (program order); or 


\begin{tabular}{lllllll}
\hline$p_{1}(\mathrm{SC}):$ & $\mathrm{w}(\mathrm{x}) 1$ & $\mathrm{r}(\mathrm{x}) 1$ & $\mathrm{w}(\mathrm{y}) 1$ & $\mathrm{r}(\mathrm{x}) 2$ & & \\
$p_{2}(\mathrm{SC}):$ & $\mathrm{w}(\mathrm{x}) 2$ & & $\mathrm{r}(\mathrm{x}) 2$ & $\mathrm{w}(\mathrm{y}) 2$ & $\mathrm{r}(\mathrm{x}) 2$ & \\
$p_{3}$ (CC): & $\mathrm{w}(\mathrm{y}) 3$ & $\mathrm{r}(\mathrm{y}) 4$ & & $\mathrm{r}(\mathrm{x}) 1$ & $\mathrm{r}(\mathrm{x}) 2$ & $\mathrm{r}(\mathrm{y}) 2$ \\
$p_{4}$ (CC): & $\mathrm{w}(\mathrm{y}) 4$ & $\mathrm{r}(\mathrm{y}) 3$ & $\mathrm{r}(\mathrm{x}) 1$ & & $\mathrm{r}(\mathrm{x}) 2$ & $\mathrm{r}(\mathrm{y}) 2$
\end{tabular}

Figure 1. Mixed Consistency Example with $O_{S C}=\left\{x_{1}, x_{2}, x_{3}, x_{4}\right\}$ and $O_{C C}=$ $\left\{y_{1}, y_{2}, y_{3}, y_{4}\right\}$

- $o p_{1} \mapsto o p_{2}$ (writes-into order); or

- there is another $o p_{3}$ such that $o p_{1} \Rightarrow o p_{3} \Rightarrow o p_{2}$

History: We define the global history (or history) of an operation set $A$, denoted as $H$, to be a collection of $A$ 's operations and the program order among those operations, i.e. $H=<A, \rightarrow>$.

\subsection{Mixed Consistency}

We say that a history $H$ is mixed-consistent if it satisfies all the following requirements:

1. SC requirement: there exists a serialization of $A^{S C}$ such that it respects the program order;

2. CC requirement: for each $\mathrm{CC}$ process $p_{i}$, there exists a serialization of $A_{\left\{p_{i}\right\}} \cup W$ such that it respects the causal order ( $W$ denotes the set of all writes).

In order to illustrate the mixed consistency model, let's consider one example. Figure 1 gives the system setting and the execution history $H$ (operations in the same column denote concurrent operations), where the notion $x_{i}$ (or $y_{i}$ ) denotes the replica of object $x$ (or $y$ ) that process $p_{i}$ has.

The history $H$ in Figure 1 is mixed-consistent because it meets all the requirements:

1. SC requirement: we know that

$$
\begin{aligned}
A^{S C}=\{ & w_{1}(x) 1, r_{1}(x) 1, r_{1}(x) 2, w_{2}(x) 2, r_{2}(x) 2, \\
& \left.r_{2}(x) 2, r_{3}(x) 1, r_{3}(x) 2, r_{4}(x) 1, r_{4}(x) 2\right\}
\end{aligned}
$$

Clearly $A^{S C}$ is well-formed. And

$$
\begin{aligned}
S=\{ & w_{1}(x) 1, r_{1}(x) 1, r_{3}(x) 1, r_{4}(x) 1, w_{2}(x) 2 \\
& \left.r_{1}(x) 2, r_{2}(x) 2, r_{2}(x) 2, r_{3}(x) 2, r_{4}(x) 2\right\}
\end{aligned}
$$

is a serialization that respects the program order of all $p_{i}(1 \leq i \leq 4)$.

2. CC requirement: we have

$$
\begin{aligned}
A_{\left\{p_{3}\right\}}= & \left\{w_{3}(y) 3, r_{3}(y) 4, r_{3}(x) 1, r_{3}(x) 2, r_{3}(y) 2\right\} \\
A_{\left\{p_{4}\right\}}= & \left\{w_{4}(y) 4, r_{4}(y) 3, r_{4}(x) 1, r_{4}(x) 2, r_{4}(y) 2\right\} \\
W= & \left\{w_{1}(x) 1, w_{2}(x) 2, w_{1}(y) 1, w_{2}(y) 2, w_{3}(y) 3,\right. \\
& \left.w_{4}(y) 4\right\}
\end{aligned}
$$

For process $p_{3}$ :

$$
\begin{aligned}
S_{3}=\{ & w_{3}(y) 3, w_{4}(y) 4, r_{3}(y) 4, w_{1}(x) 1, r_{3}(x) 1, \\
& \left.w_{2}(x) 2, r_{3}(x) 2, w_{1}(y) 1, w_{2}(y) 2, r_{3}(y) 2\right\}
\end{aligned}
$$

is the serialization that respects the causal order.

For process $p_{4}$ :

$$
\begin{aligned}
S_{4}=\{ & w_{4}(y) 4, w_{3}(y) 3, r_{4}(y) 3, w_{1}(x) 1, r_{4}(x) 1, \\
& \left.w_{2}(x) 2, r_{4}(x) 2, w_{1}(y) 1, w_{2}(y) 2, r_{4}(y) 2\right\}
\end{aligned}
$$

is the serialization that respects the causal order.

\section{A Mixed Consistency Protocol}

To design brand new protocols to ensure either SC or $\mathrm{CC}$ is not our purpose. Instead, we show in this paper that we can combine existing SC and CC protocols together to meet mixed consistency requirements. We choose two wellstudied protocols in the literature to achieve this goal.

SC: Home-based Protocol We choose home-based protocol [6] to ensure SC requirements. A designated home node is associated with every object that has a SC replica to coordinate access to such replicas. A node with SC replica must acquire a token prior to access. The home node keeps track of what nodes can read or write the object's replicas and grants READ/WRITE_TOKEN to the nodes that want to read/write this object. The READ_TOKEN is shared by multiple readers, while the WRITE_TOKEN does not coexist with the READ_TOKEN and can only be issued to one writer at a time. Tokens are issued based on a First-ComeFirst-Serve order observed by the home node. Currently, the location of the home node is randomly assigned in our protocol, although results in $[7,19,21]$ suggest that random assignment may have a negative impact on the performance of home-based protocols. We can employ a "smart" home node assignment when an application profile is available.

CC: Causal Memory Protocol We choose vector clock based causal memory protocol [2] to ensure $\mathrm{CC}$ requirements are met. When a write operation is performed, a new value along with the local clock will be disseminated to other replicas. The receiver applies the new value when all the "causally preceding" values have arrived and been applied, which is determined based on the receiver's local vector clock and the clock value that comes with the new object value. In this protocol, the dissemination process can be done in background. Therefore, write operations do not block and return immediately. 


\begin{tabular}{|c|c|}
\hline \multicolumn{2}{|l|}{ INITIALIZATION } \\
\hline NAME & FUNCTIONALITY \\
\hline init() & Initialize local data structures such as timestamps and queues. \\
\hline \multicolumn{2}{|c|}{ APPLICATION INTERFACE (invoked by upper layer) } \\
\hline $\operatorname{read}(\mathrm{x})$ & Return the value $\mathrm{v}$ of object $\mathrm{x}$. \\
\hline write $(\mathrm{x}, \mathrm{v})$ & Write new value $\mathrm{v}$ to object $\mathrm{x}$. \\
\hline \multicolumn{2}{|c|}{ LOCAL FUNCTIONS (invoked by local application interfaces) } \\
\hline write_miss $(\mathrm{x}, \mathrm{v})$ & Request a new WRITE_TOKEN from the home node and complete the local write. \\
\hline read_miss(x) & Request a new READ_TOKEN from the home node and pull new value from the latest writer if necessary. \\
\hline \multicolumn{2}{|c|}{ SYNCHRONOUS COMMUNICATION PRIMITIVES (invoked by remote nodes, block before return) } \\
\hline value_request(y) & Return the latest value of y (and associated timestamps, if necessary) to the caller node. \\
\hline write_token_request(y) & Return the new WRITE_TOKEN to the caller node after revoking all other tokens. \\
\hline read_token_request(y) & Return the new READ_TOKEN to the caller node after revoking other's WRITE_TOKEN, if any. \\
\hline write_token_revoke(y) & Delete local WRITE_TOKEN. \\
\hline read_token_revoke(y) & Delete local READ_TOKEN. \\
\hline \multicolumn{2}{|c|}{ ASYNCHRONOUS COMMUNICATION PRIMITIVES (keep running forever, monitoring queues) } \\
\hline send_daemon() & Keep running forever; send msgs to selected destinations from output queue oqueue. \\
\hline receive_daemon() & Keep running forever; receive msgs from the input queue iqueue, and apply new values if necessary. \\
\hline
\end{tabular}

Figure 2. Categorized interfaces of mixed consistency protocol

\subsection{Challenges for Mixed Consistency}

We give our mixed consistency protocol in Figure 6. The protocol interfaces are summarized in Figure 2, which will be explained later in the paper to help understand the protocol. Before that, we want to address several problems of integrating the SC and CC protocols together in order to implement the mixed consistency model.

Possible Causal Order Violation: In our model, a process can have both SC and CC replicas. If we simply run the two protocols to maintain replicas based on their type, we could have a potential CC violation. For instance, suppose there are two processes, namely $p_{1}$ (SC) and $p_{2}$ (CC). Each has a SC copy of $o_{1}$ and a CC copy of $o_{2} \cdot p_{1}$ writes $o_{2}$ first and then writes $o_{1} \cdot p_{2}$ reads $o_{1}$ and then $o_{2}$. The home-based protocol can guarantee that $p_{2}$ reads what $p_{1}$ writes to $o_{1}$. But when $p_{2}$ reads $o_{2}$, it might get the "old" value because $p_{1}$ 's new value for $o_{2}$ is possibly still on the way because of the background dissemination of the causal memory protocol. We overcome this obstacle by delaying $\mathrm{CC}$ reads (setting the replica as NOT READABLE) if a potential $\mathrm{CC}$ violation is possible. In Figure 6, function read_miss() evaluates the vector clock returned by process $p_{k}$ and sets any CC object as NOT READABLE if a causal order violation is possible as described above. New CC values will eventually arrive (please refer to Section 4.4) so the delayed CC reads will eventually return with the correct value. In Figure 6, function receive daemon() resets the object as READABLE when it receives a new CC value.

Possible Vector Clock Error: When a SC process writes a SC object, the new value will be disseminated to the $\mathrm{CC}$ replicas held by other processes. The receiver needs a correct vector clock in the message to order the update to the $\mathrm{CC}$ replica (i.e. when to apply the new value). Therefore, all SC replicas should maintain a "correct" vector clock associated with them, which in turn requires that when a CC pro- cess writes a CC object, although the value does not propagate to SC replicas (otherwise violates Rule 1), the vector clock does. Our design enforces the dissemination of vector clocks as required. In Figure 6, function send_daemon() sends the message to all processes (both $\mathrm{SC}$ and $\mathrm{CC}$ ) when the local writer $p_{i}$ is a $\mathrm{CC}$ process. Please note that this does not violate our access constraints because the function receive_daemon () at the receiver's side only applies the value to the local copy when the receiver is a CC process. If the receiver is a SC process, only the vector clock is used to keep the local vector clock correct. The $\mathrm{CC}$ value embedded in the message is discarded.

\subsection{Protocol Interfaces}

The protocol interface is shown in Figure 2, where we categorize the functions into initialization, application interface, local functions, synchronous and asynchronous communication primitives. We use the term "synchronous communication" to refer to blocking, RPC style communication. It is performed through Remote Method Invocation (RMI). We use "asynchronous communication" to refer to non-blocking, send/receive daemon style communication. Our protocol does not place any specific requirements on how to perform asynchronous dissemination. Various multicast or rumor-spreading based techniques can be used as the communication layer support for our protocol. We are exploring an adaptive communication layer under our protocol, featuring better performance and bandwidth utilization in a heterogeneous environment.

In our protocol, read $(x)$ and write $(x, v)$ are the interfaces exposed to the applications. These two functions first evaluate if the constraints in Table 1 are violated. If yes, an exception will be thrown. For a SC replica write shown in Figure 3 and 4, write $(x, v)$ triggers a write_miss $(x, v)$ call if a WRITE_TOKEN is missing, 
which in turn asks the home node for a write token by calling write_token_request $(x)$. Old or conflicting tokens are revoked by write/read_token_revoke $(x)$. A SC replica read, shown in Figure 5, can triggers a read miss $(x)$ if a READ_TOKEN is missing. When the home node issues the READ_TOKEN, it also tells the reader where the latest copy is (the latest writer) and the reader calls value request $(x)$ to fetch the copy. Vector clocks are also returned along with the latest copy by the latest writer in order to ensure the cross SC/CC causal relationship is correct.

For a CC replica write, a message is constructed and inserted into the outgoing queue (oqueue). Function send_daemon() will eventually send out the message and the destination side receive_daemon() will eventually receive it. A CC replica read is returned immediately if the replica is READABLE. As restricted by the constraints of Table 1, writes to a CC replica will not be propagated to SC replicas. However, we do propagate the meta data (vector clock, to be specific) from $\mathrm{CC}$ replicas to $\mathrm{SC}$ replicas when a write to CC replica happens. This ensures that the vector clocks on SC replicas can correctly capture the causal relationship even though they do not share the $\mathrm{CC}$ values.

\subsection{Correctness of the Protocol}

The protocol given in Figure 6 is correct because we can show that both $\mathrm{SC}$ and CC requirements are met in this protocol (due to page limitation, we only give a sketch of the proof in this paper).

(1) If there are only $S C(C C)$ replicas in the system, the protocol behaves the same as the home-based protocol [6] (causal memory protocol [2]) does. Both SC and $\mathrm{CC}$ requirements are met.

(2) If there are mixed SC and CC replicas in the system, the SC requirements are not violated because the definition of access constraints isolates the writes to $\mathrm{CC}$ replicas from $\mathrm{SC}$ replicas. These writes, which are not sequentially ordered, are not visible to $\mathrm{SC}$ replicas. Now we are trying to argue that $\mathrm{CC}$ requirements are not violated either. Let's suppose $\mathrm{CC}$ requirements are violated. There are two possibilities: either the causal order among CC objects is broken, or the causal order among SC and CC objects is broken. The correctness of the causal memory protocol guarantees that the first case does not happen. Our solutions proposed in Section 4.1 prevent the second case from happening. So we can be sure that causal order is correctly maintained in our protocol. Therefore, both SC and CC requirements are met.

\subsection{Communication and Host Failures}

In this paper, we mainly focus on introducing the mixed consistency model and the protocol described in Figure 6 assumes a failure free environment for simplicity reasons. However, our protocol can be extended to handle communication and node failures. In a fault-tolerant version of the mixed consistency protocol that we are developing, we assume omission failure of communication channels and failstop failures of nodes where the processes execute. The omission failure is tolerated by the re-transmission support of the communication layer which our protocol is built upon. When a node fails, all the replicas maintained by that node fail too. The CC protocol used in our MC implementation can be easily extended to tolerate fail-stop failures, while the home-based SC protocol cannot. When object $x$ 's home node $D_{x}$ fails, all read and write operations on $x$ block and $x$ becomes inaccessible. However, our mixed consistency model provides a new way to address fail-stop failure handling through replica downgrading, which allows computation to continue at a lower consistency level when failures happen.

The downgrading process happens when the system detects that $D_{x}$ has failed. The system will then downgrade all the $S C$ replicas of $x$ maintained by non-faulty nodes to $\mathrm{CC}$ copies. During the downgrading process, all the write operations on all replicas will be frozen in order to maintain correct causal relationship. The downgrading procedure can be summarized in three steps: 1) When a process detects that $D_{x}$ has failed, it sends out a DOWNGRADING_START message to all the nodes in the system, initializing the start of downgrading (Duplicated DOWNGRADING_START messages will be ignored). 2) The actual downgrading process starts when the system has applied all outstanding writes. During the downgrading process, all new write requests will be delayed until the process is finished. Each node that has a $S C$ copy of $x$ will downgrade it to CC. 3) After a node finishes its downgrading, a LOCAL_DOWNGRADING_FINISH message will be sent to all other nodes. When a node receives all the LOCAL_DOWNGRADING_FINISH messages, the computation will continue with only CC copies of object $x$ in the system. Since an application may want stronger consistency, we are also exploring protocols that can upgrade replicas after a node recovers from failures.

\section{Analysis}

A simple system is used to analyze the network traffic and timeliness of the mixed consistency protocol. It consists of $n+m$ processes ( $n \mathrm{SC}$ processes and $m$ CC processes) sharing one object $x$. We assume that the average one-way message transmission time is $t$. The dissemination is done by a tree-based protocol, with a fan-out factor of $f$. For example, the average time for a process to disseminate a new value to $m$ processes is $\left(\log _{f} m\right) t$. We estimate the network traffic by the number of messages generated for different 


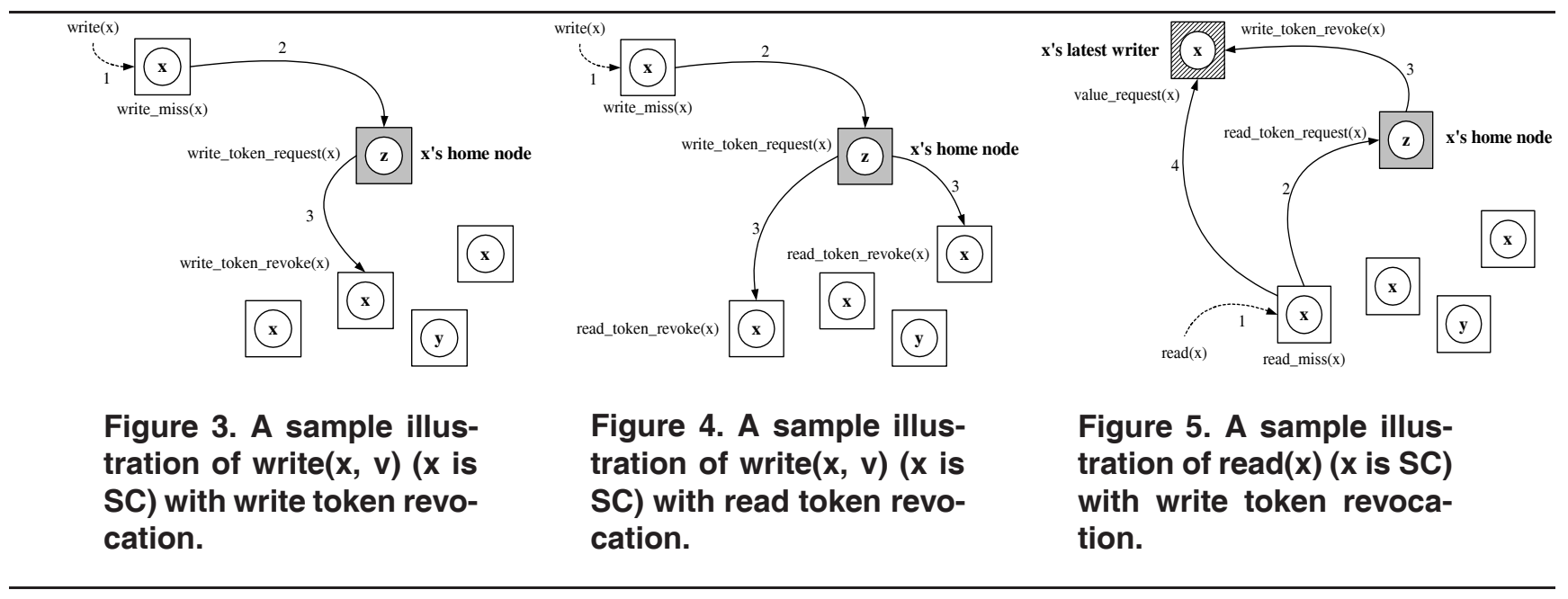

operations. The timeliness of read operation is estimated by the time it takes to return a value, the timeliness of write operation is estimated by the time it takes for the rest of the system to become aware of its new value. We compare the mixed consistency protocol against SC consistency and CC consistency implementations in Figure 7.

SC consistency (home-based protocol implementation) Let's assume $x$ 's home node is $p_{0}$.

- READ: Let's further assume process $p_{i}\left(\neq p_{0}\right)$ does not have $x$ 's READ_TOKEN and $p_{j}\left(\neq p_{0}\right)$ is last writer of $x$, so a read request at $p_{i}$ will generate additional control messages for requesting/revoking tokens.

- Suppose there is no other readers between $p_{j}$ 's write and $p_{i}$ 's read, the number of messages for $p_{i}$ to return the latest value of $x$ is 6 (1 READ_TOKEN_REQUEST from $p_{i}$ to $p_{0}, 1$ WRITE_TOKEN_REVOKE from $p_{0}$ to $p_{j}, 1$ VALUE_REQUEST from $p_{i}$ to $p_{j}$, and 3 REPLY messages for each of these messages). The total time for $p_{i}$ to return a new value is $6 t$.

- Suppose there are other readers that read $x$ 's value before $p_{i}$, the number of messages will be reduced to 4 ( $p_{0}$ does not need to revoke $p_{j}$ 's WRITE_TOKEN). And the total time is $4 t$.

- WRITE: Let's assume process $p_{i}\left(\neq p_{0}\right)$ does not have $x$ 's WRITE_TOKEN. There are two possibilities: a process $p_{j}\left(\neq p_{0}\right)$ holds $x$ 's WRITE_TOKEN, or there are $k$ processes holding $x$ 's READ_TOKEN. The total number of messages for $p_{i}$ to complete a write operation is 2 and $2(k+1)$ respectively. Therefore, the total time for $p_{i}$ 's write is either $2 t$ or $2(k+1) t$.

- For both READ and WRITE, if $p_{i}$ already has the proper token, no messages will be generated. Thus we say that the communication time each operation takes is 0 .

\section{CC consistency (vector clock based protocol)}

- READ: Since there is no communication involved for serving read requests, no messages will be generated. And the time to complete a read request is 0 .

- WRITE: A new object value (together with vector clock) needs to be disseminated to all other processes, when a write request is serviced. So a total number of $n+m-1$ messages will be generated. It takes an average of $t\left(\log _{f}(n+m-1)\right)$ for this new object value to be disseminated to all other processes, although the write operation itself immediately returns before communication completes.

\section{Mixed consistency}

- SC READ: Same as READ in SC consistency, i.e. 4 or 6 messages and $4 t$ or $6 t$ time.

- SC WRITE: Similar as WRITE in SC consistency, except that $m$ additional dissemination messages are generated because the writer needs to disseminate the new value to all $\mathrm{CC}$ replicas as well. Therefore, the total number of messages is either $2+m$ or $2(k+1)+m$. And the total execution time of write is $2 t$ or $2(k+1) t$, the dissemination time is $\log _{f} m$.

- For both $S C R E A D$ and $S C W R I T E$, if $p_{i}$ already has the proper token, no control messages will be generated ( $m$ additional dissemination messages are still generated for $S C$ WRITE, taking $t\left(\log _{f} m\right)$ time to be disseminated) and both operations immediately return with 0 communication time. 
Assume there are $\mathrm{N}$ processes in the system,

i.e. $p_{1}, \ldots, p_{N}$.

0 : array of $\mathrm{M}$ objects. $\mathrm{x}$ : object name.

i.e. $O[x]$ contains value $v$ of object $x$.

init ()

(rinitializing the meta data

for every CC object $\mathrm{x}$ do set $x$ as READABLE

for every object $\mathrm{x}$ do

for $j=1$ to $\mathrm{N}$ do

$t_{x}[j]=0 ; / /$ initialize the timestamp

oqueue $=\langle>$
iqueue $=\langle>$

write (x, v)

/write new value $v$ to object $x$

if $\mathrm{x} \in \mathrm{SC}$ and $p_{i} \in S C$

if $p_{i}$ has $x^{\prime} \mathrm{S}$ WRITE_TOKEN

$t_{x}[i]=t_{x}[i]+1$

$\mathrm{O}[\mathrm{x}]=\mathrm{V}$;

enqueue (oqueue, $\langle i, x, v, t>$ )

else write_miss ( $\mathrm{x}, \mathrm{v})$

else if $x \in C C$

$t_{x}[i]=t_{x}[i]+1$.

$\mathrm{O}[\mathrm{x}]=\mathrm{V}$

enqueue (oqueue, $<i, x, v, t>$ )

else throw WRITE_EXCEPTION;

$\underline{\operatorname{read}(\mathrm{x})}$

l/read object $x^{\prime}$ s value

if $x \in C C$ and $p_{i} \in C C$

if $x$ is NOT READABLE

wait until $x$ is READABLE; return $\mathrm{O}[\mathrm{x}]$

else if $\mathrm{x} \in \mathrm{SC}$

if $p_{i}$ has $\mathrm{x}^{\prime} \mathrm{s}$ READ_TOKEN

return $\mathrm{O}[\mathrm{x}]$

else return read_miss $(x)$

else throw READ_EXCEPTION;

write_miss $(\mathrm{x}, \mathrm{v})$

//wait for a WRITE_TOKEN from $x^{\prime}$ s home node $p_{j}$ calls $p_{j} \cdot$ write_token_request (x)

set ' $p_{i}$ has $\mathrm{x}^{\prime} \mathrm{s}$ WRITE_TOKEN' ';

$t_{x}[i]=t_{x}[i]+1 ;$

$\mathrm{O}[\mathrm{x}]=\mathrm{v} ;$

enqueue (oqueue, $\langle i, x, v, t>$ ) :

\section{read_miss ( $\mathrm{x}$ )}

lwait for a READ_TOKEN from $x$ 's home node $p_{j}$

call $p_{k}=p_{j}$. read_token_request (x);

//assume $p_{k}$ has the latest value of $x$

set ' $p_{i}$ has $\mathrm{x}$ 's READ_TOKEN',

if $p_{k}$ is null

return $\mathrm{O}[\mathrm{x}]$

else

call $<\mathrm{O}[\mathrm{x}], \mathrm{s}>=p_{k}$. value_request $(\mathrm{x})$

for any $\mathrm{CC}$ object $z$ do

if $\left(\exists j \neq i: \mathrm{s}_{z}[j]>\mathrm{t}_{z}[j]\right)$

set $z$ as NOT READABLE;

if $\left(s_{x}[i]>t_{x}[i]\right)$

$\mathrm{t}_{x}[\mathrm{i}]=\mathrm{s}_{x}[\mathrm{i}]$

return $\mathrm{O}[\mathrm{x}]$ :

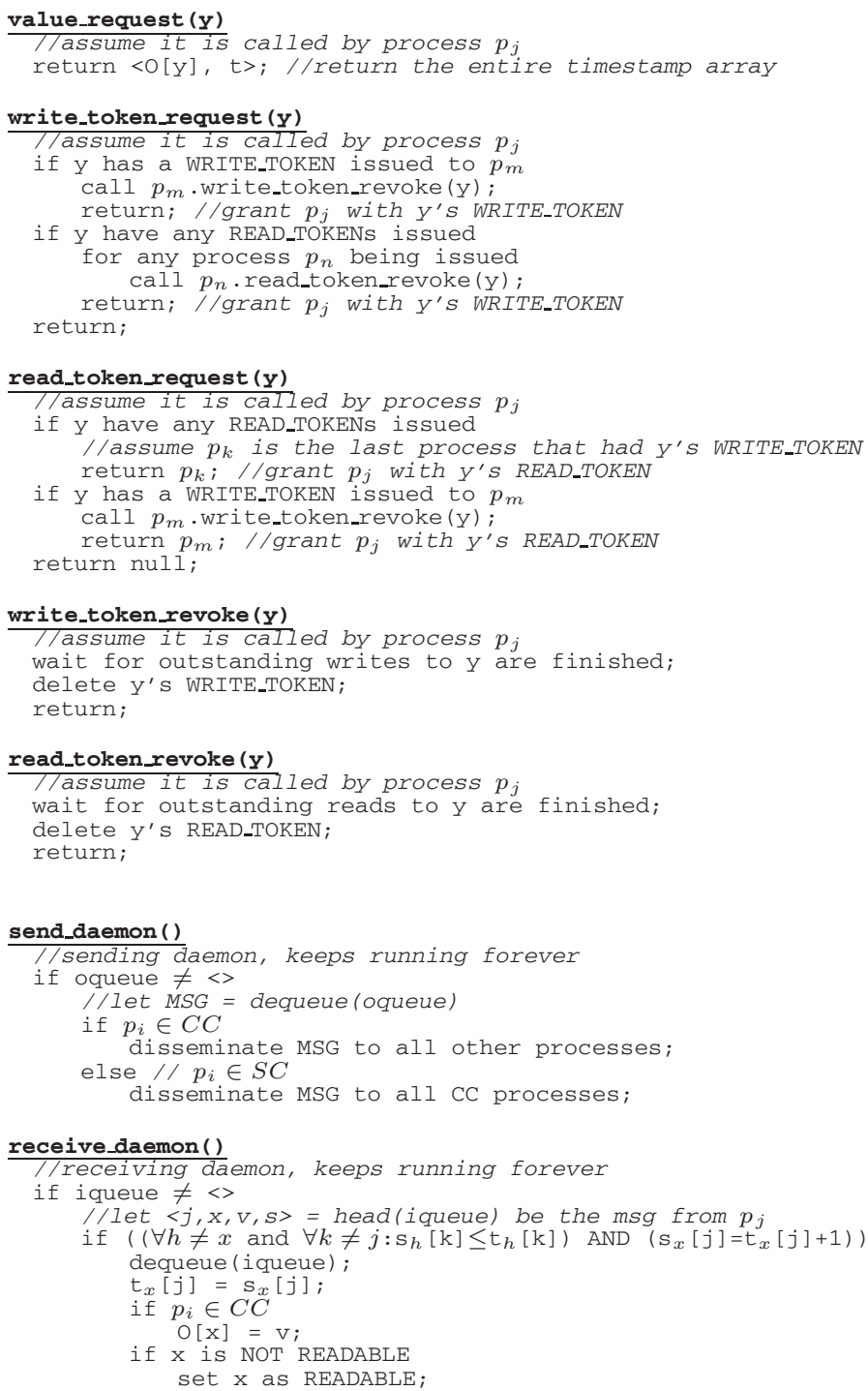

Figure 6. The Mixed Consistency Protocol - at process $p_{i}$

\begin{tabular}{|l|l|l|l|}
\hline \multirow{2}{*}{ READ in SC } & Network Traffic & Time \\
\cline { 2 - 4 } & reader has token & 0 & 0 \\
\cline { 2 - 4 } & reader does not have token & 4 or 6 & $4 \mathrm{t}$ or $6 \mathrm{t}$ \\
\hline \multirow{2}{*}{ WC READ in MC } & reader has token & 0 & 0 \\
\cline { 2 - 4 } & reader does not have token & 4 or 6 & $4 \mathrm{t}$ or $6 \mathrm{t}$ \\
\cline { 2 - 4 } & writer has token & 0 & 0 \\
\cline { 2 - 4 } & writer does not have token & 2 or $2(\mathrm{k}+1)$ & $2 \mathrm{t}$ or $2(\mathrm{k}+1) \mathrm{t}$ \\
\hline \multirow{2}{*}{ SC WRITE in MC } & writer has token & $\mathrm{m}$ & 0 execution, $\log _{f} m$ dissemination \\
\cline { 2 - 4 } & writer does not have token & $2+\mathrm{m}$ or $2(\mathrm{k}+1)+\mathrm{m}$ & $2 \mathrm{t}$ or $2(\mathrm{k}+1) \mathrm{t}$ execution, $\log _{f} m$ dissemination \\
\hline READ in CC & - & 0 & 0 \\
\hline CC READ in MC & - & 0 & 0 \\
\hline WRITE in CC & - & $\mathrm{n}+\mathrm{m}-1$ & 0 execution, $\log _{f}(n+m-1)$ dissemination \\
\hline CC WRITE in MC & - & $\mathrm{n}+\mathrm{m}-1$ & 0 execution, $\log _{f}(n+m-1)$ dissemination \\
\hline
\end{tabular}

Figure 7. Analysis of mixed consistency protocol 
- CC READ: Same as READ in CC consistency.

- CC WRITE: Similar as WRITE in CC consistency, $m-1$ dissemination messages are generated. In order to prevent "possible vector clock error", the writer needs to send its vector clock to all SC processes. So $n$ control messages are generated. A total number of $n+m-1$ messages will be generated. The time is also the same as WRITE in CC consistency.

To summarize, the network traffic and timeliness of SC, $\mathrm{CC}$ and $\mathrm{MC}$ models are shown in Figure 7. It is clear to see that the performance of mixed consistency protocol is almost the same as SC and CC, depending on what tag the reader/writer has. The overall performance of an MC system is determined by how many $\mathrm{SC} / \mathrm{CC}$ replicas are there in the system.

\section{Related Work}

Agrawal et. al. first introduced the term mixed consistency in [1] to refer to a parallel programming model for distributed shared memory systems, which combines causal consistency and PRAM [13] consistency. Four kinds of explicit synchronization operations: read locks, write locks, barriers and await operations are provided in their model. In this paper, we use the same term to refer to a new consistency model combining sequential consistency and causal consistency. The access constraints we propose represent a more general approach: PRAM consistency can be integrated into our mixed consistency model with little effort.

Attiya and Friedman introduced the concept of hybrid consistency in [4]. In this model, all read and write operations on shared objects are categorized as either strong or weak. All processes agree on a sequential order for all strong operations, and on the program order for any two operations issued by the same process in which at least one of them is strong. It does not guarantee any particular order of any two weak operations between two strong operations. In our model, an operation is weak or strong depending on whether it is executed with a strong or weak replica. Thus, both strong and weak operations can not be executed on a replica by the same process. We define access constraints to develop the mixed consistency model where we do not assume that strong operations can be used to establish order among weak operations when necessary.

Fernandez, Jimenez, and Cholvi proposed a simple algorithm to interconnect two or more causal consistency systems into one causal consistency system through gates in [8]. They further defined a formal framework to describe the interconnection of distributed shared memory systems in [9] and showed that only fast memory models can be interconnected, where read and write operations return immediately after only local computations (i.e. no global syn- chronization, which is required to implement SC systems). In our mixed consistency model, we take a different access constraints based approach to combine $\mathrm{SC}$ and $\mathrm{CC}$ together. We do not try to interconnect SC and CC through gates, where all the communications between two systems must flow through. Therefore, our result does not contradict theirs.

Yu and Vahdat presented a conti-based continuous consistency model for replicated services in paper [20]. In their model, applications use three measurements of numerical error, order error and staleness as bounds to quantify their relaxed consistency requirements. The authors used relaxed consistency model with bounded inconsistency to balance the tradeoffs between performance, consistency and availability. By exploring application and system resource heterogeneity, we also suggest that replicated distributed services should be able to support more than just strong consistency. However, instead of relaxing strong consistency requirements, we believe that certain applications can benefit from having both strongly and weakly consistent replicas at the same time.

Raynal and Mizuno survey many consistency models for shared objects in [15]. They particularly emphasize linearizability, SC, hybrid consistency and CC. Raynal and Schiper [16] show that SC is strictly stronger than CC by showing that CC + "total order on all writes on all objects" $=\mathrm{SC}$. Based on the results of these two papers, we choose $\mathrm{SC}$ and $\mathrm{CC}$ as the example of strong and weak consistency to implement the mixed consistency model in this paper.

Two orthogonal dimensions of consistency, timeliness and ordering, are explored in [3]. Ordering determines the value that should be applied/returned by the consistency protocols. Sequential consistency [12], [5], [16] requires all operations appear to be executed in a serial order that respects the "read-from" ordering and "process" ordering. Causal consistency [2] is a weaker form of consistency model because it does not require all replicas to agree on a unique execution history. There are various other consistency models (e.g. lazy release consistency [10] and Bayou session guarantees [18]), which explore different aspects (e.g. synchronization, transaction) of distributed computation and how extra facility can be used to enhance consistency models. Timeliness decides how fast the proper value should be applied/returned. Maintaining web content consistency [14] and $\delta$-time consistency [17] suggest that the value returned should be at most $\delta$ time unit "old". [11] presents an efficient implementation of timed consistency based on combined "push" and "pull" techniques. Our work fits into the operation ordering dimension of consistency model. The mixed consistency model tries to combine different order guarantees together to satisfy application needs in wide area heterogeneous environment. 


\section{Conclusion}

In this paper, we propose a mixed consistency model to combine existing strong (SC) and weak (CC) consistency models together to meet the heterogeneity challenge for large scale distributed shared memory systems. Our model defines new SC and CC requirements on subsets of replicas shared by different processes. We propose access constraints as the base to implement our mixed consistency model. We make minor modifications to two existing protocols and combine them together to implement the mixed consistency model. We show that the result protocol satisfies all the requirements we define.

The access constraints based approach we proposed in this paper is not limited to combining just SC and CC. Currently we are exploring the combination of other consistency schemes under similar access constraints. We'd like to implement a fault-tolerant version of our protocol in order to better meet the application needs under real life conditions. And as an extension of our work, we are building our protocols on top of an adaptive update dissemination framework, which can provide better performance and bandwidth utilization in a heterogeneous environment.

\section{Acknowledgements}

We thank anonymous reviewers and our sherperd Dr. Neeraj Suri. Their comments have greatly improved this paper.

\section{References}

[1] D. Agrawal, M. Choy, H. V. Leong, and A. K. Singh. Mixed consistency: a model for parallel programming (extended abstract). In The 13th Annual ACM Symposium on Principles of Distributed Computing (PODC), 1994.

[2] M. Ahamad, G. Neiger, J. E. Burns, P. W. Hutto, and P. Kohli. Causal memory: Definitions, implementation and programming. Distributed Computing, 9:37-49, 1995.

[3] M. Ahamad and M. Raynal. Ordering vs timeliness: Two facets of consistency? Future Directions in Distributed Computing, 2003.

[4] H. Attiya and R. Friedman. A correctness condition for highperformance multiprocessors. In Proc. of the 24th ACM Symposium on Theory of Computing (STOC), 1992.

[5] H. Attiya and J. L. Welch. Sequential consistency versus linearizability. ACM Transactions on Computer Systems, 1994.

[6] J. Carter, J. Bennett, and W. Zwaenepoel. Implementation and performance of munin. In Proceedings of the 13th ACM Symposium on Operating Systems Principles (SOSP), 1991.

[7] A. Cox, E. de Lara, and W. Z. Y. C. Hu. A performance comparison of homeless and home-based lazy release consistency protocols for software shared memory. In Proc. of the 5th IEEE Symp. on High-Performance Computer Architecture (HPCA-5), 1999.
[8] A. Fernandez, E. Jimenez, and V. Cholvi. On the interconnection of causal memory systems. In Proc. of the 19th ACM Symposium on Principles of Distributed Computing (PODC), July 2000.

[9] E. Jimenez, A. Fernandez, and V. Cholvi. Decoupled interconnection of distributed memory models. In Proc. of the 7th International Conference on Principles of Distributed Systems (OPODIS 2003), December 2003.

[10] P. Keleher, A. L. Cox, and W. Zwaenepoel. Lazy release consistency for software distributed shared memory. In Proc. of the 19th Annual Int'l Symp. on Computer Architecture (ISCA'92), pages 13-21, 1992.

[11] V. Krishnaswamy, M. Ahamad, M. Raynal, and D. Bakken. Shared state consistency for time-sensitive distributed applications. In 21th International Conference on Distributed Computing Systems (ICDCS), 2001.

[12] L. Lamport. How to make a multiprocessor computer that correctly executes multiprocess programs. IEEE Transactions on Computers, C28(9):690-691, 1979.

[13] R. Lipton and J. S. Sandberg. Pram: A scalable shared memory. Technical Report CS-TR-180-88, Princeton University, Dept. of Computer Science, 1988.

[14] C. Liu and P. Cao. Maintaining strong cache consistency in the world-wide web. In International Conference on Distributed Computing Systems (ICDCS), 1997.

[15] M. Raynal and M. Mizuno. How to find his way in the jungle of consistency criteria for distributed objects memories (or how to escape from minos'labyrinth). In Proc. of the IEEE International Conference on Future Trends of Distributed Computing Systems, September 1993.

[16] M. Raynal and A. Schiper. From causal consistency to sequential consistency in shared memory systems. In 15th Conference on Foundations of Software Technologies and Theoretical Computer Science, pages 180-194, Bangalore, India, 1995. Springer-Verlag.

[17] A. Singla, U. Ramachandran, and J. K. Hodgins. Temporal notions of synchronization and consistency in beehive. In ACM Symposium on Parallel Algorithms and Architectures, pages 211-220, 1997.

[18] D. B. Terry, M. M. Theimer, K. Peterson, A. J.Demers, M. J. Spreitzer, and C. H. Hauser. Managing update conflicts in bayou, a weakly connected replicated storage system. In Proceedings of the 15th ACM SOSP, 1995.

[19] B.-H. Yu, Z. Huang, S. Cranefield, and M. Purvis. Homeless and home-based lazy release consistency protocols on distributed shared memory. In 27th Australasian Computer Science Conference (ACSC'04), 2004.

[20] H. Yu and A. Vahdat. Design and evaluation of a conitbased continuous consistency model for replicated services. In ACM Transactions on Computer Systems (TOCS), 2002.

[21] Y. Zhou, L. Iftode, and K. Li. Performance evaluation of two home-based lazy release consistency protocols for shared memory virtual memory systems. In Proc. of the 2nd Symp. on Operating Systems Design and Implementation (OSDI'96), pages 75-88, 1996. 